\title{
Photoemission spectra of indium selenide
}

\author{
V.M. Katerynchuk, M.Z. Kovalyuk, M.V. Tovarnitskii \\ I. Frantsevich Institute for Problems of Material Science, NAS of Ukraine, Chernivtsi Department \\ 5, Iryna Vilde str., 58001 Chernivtsi, Ukraine \\ Phone: (0372) 525155; fax: (03722) 36018; e-mail: chimsp@unicom.cv.ua
}

\begin{abstract}
For layered InSe crystals photoluminescence spectra were investigated and the corresponding radiative transitions were analyzed. It was found that along with the bandto-band transitions the radiative ones with participation of impurity levels plays a substantial role in the light emission spectrum of the semiconductor. This fact is confirmed by the intensities of the radiative bands for the impurity level $-c(v)$-band transitions and the donor-acceptor recombination. The temperature dependences of the spectrum in the range of 100 to $300 \mathrm{~K}$ have also enabled to ascertain the dynamics of these radiative transitions. At the temperatures 180 to $300 \mathrm{~K}$, the bands associated with the indirect transitions involving indirect free excitons are more intensive. At $100 \ldots 180 \mathrm{~K}$, the intensity of the bands corresponding to the direct transitions with participation of direct free excitons increases. We have determined the energies of the observed photoluminescence bands, and the band diagram of the corresponding radiative transitions in InSe has been built.
\end{abstract}

Keywords: InSe, layered crystal, photoluminescence, energy diagram.

Manuscript received 10.10.06; accepted for publication 23.10.06.

\section{Introduction}

InSe crystals are an interesting object for optical investigations including photoluminescence (PL) measurements. The layered crystal structure favours to this study allowing to easily prepare samples of arbitrary thickness by cleaving them from an ingot. Their mirrorlike surfaces are practically ideal with a normal parallel to the crystallographic $C$ axis. A variety of electron transitions determines a complicated structure of PL spectra due to the presence of energy levels of various nature in the InSe energy gap [1-8]. So, InSe is an indirect semiconductor [9]. The presence of large concentrations of native donors and acceptors, which follows from compensative character of its electric properties [10], results in the existence of corresponding levels. The strong electron-hole interaction in the crystal promotes the appearance of additional levels. It causes the creation of both free and bound excitons.

An analysis of the PL spectra of layered InSe crystals shows that different authors have found various PL spectra structure and propose various interpretations for them. It is necessary to specify that InSe can be grown from both stoichiometric and nonstoichiometric composition of melts [11]. It also can affect the quality of the samples and the structure of PL spectra. Some authors observed the dependence of luminescence intensity on a method of sample preparation [3]. In a majority of the papers, PL spectra were investigated at the temperatures $4.2-77 \mathrm{~K}$ due to their weak intensity at higher temperatures.

However, we have observed the intensive PL spectra of InSe even at the room temperature. Therefore, the task of this work was to identify radiative transitions and to study their temperature dynamics.

\section{Experimental}

Indium monoselenide single crystals were grown using the Bridgman method from a nonstoichiometric melt of the components. This method allows to grow crystals with a high degree of perfection. They were obtained as ingots of 5-6 cm long and $12-14 \mathrm{~mm}$ in diameter. The ingots were cut on disks of $4-5 \mathrm{~mm}$ in height, from which plane-parallel samples were cleaved. 200-300 $\mu \mathrm{m}$ plates for our measurements were chosen in such a way to avoid their possible deformations and surface scratches.

The PL spectra were measured using a MDR-3 diffraction monochromator. Its resolution was chosen in such a way to have the best correlation between PL signal intensity and resolution value. Light-emitting GaP diodes were used as a source of continuous excitation with a radiation wave length of $0.55 \mu \mathrm{m}$. The 
temperature measurements of the PL spectra were carried out in the range of 100 to $300 \mathrm{~K}$ in a cryostat with an accuracy of temperature stabilization of $\pm 0.1 \mathrm{~K}$. The spectra measurements were carried out by $30 \mathrm{~K}$ steps. The PL spectra were registered using a calibrated $\mathrm{Si}$ photodiode. All the spectra were corrected with respect to the spectral sensitivity of the photodiode. The area of the light spot on the sample surface was about $0.5 \mathrm{~mm}^{2}$.

\section{Results and discussion}

A typical PL spectrum of InSe at various temperatures is shown in Fig. 1. It consists of many bands with varying intensities. At room temperature, these bands are marked by letters A, B, C, D, E $\mathrm{E}_{1}$, and E. At certain temperatures, the spectra are not shown owing to negligible changes in intensities of the radiation bands and their insignificant energy shift with lowering the temperature. The bands $\mathrm{A}, \mathrm{B}$, and $\mathrm{C}$ are caused by the radiative transitions with participation of donor and acceptor levels, because these bands are located at the longwave spectral edge The peaks of energy positions of these bands and their intensities do not have pronounced temperature dependence (Fig. 2), they also resides at these levels. The electron transitions from the donor level $E_{D}$ to the valence band correspond to the most intensive band (C) of three observed ones (Fig. 3). The transitions from the conduction band to the acceptor level $E_{A}$ correspond to the band B (Fig. 3). Such identification of the bands is caused by the fact that compensated InSe has the electron conductivity. A partial compensation of the donor levels is indicative of the less concentration of the acceptor levels and, therefore, the transitions with participation of the acceptor levels $E_{A}$ have the lower intensity ( $\mathrm{B}$ band) in comparison to $\mathrm{C}$ band. The transitions from the donor to acceptor levels correspond to the weakest band A.

The band D (that is the most intensive at $T=295 \mathrm{~K}$ from all the bands) corresponds to the indirect band-toband transitions. Such transitions occur with participation of phonons. According to [12], the D band intensity can be described by the equation

$I(h v) \approx v^{2}\left(h v-E_{g}^{i}\right)^{2} \exp \left[-\frac{\left(h v-E_{g}^{i}\right)}{k T}\right]$,

where $v$ is the light frequency, $h$ is the Plank constant, $E_{g}^{i}$ is the indirect bandgap of InSe, $k$ is the Boltzmann constant, $T$ is the absolute temperature. The band calculated according to relation (1) is shown in Fig. If by open circles. As one can see, there is a good agreement between the theoretical and experimental curves. From comparison of these curves, we have estimated that the value of $E_{g}^{i}$ at $100 \mathrm{~K}$ is equal to $1.27 \mathrm{eV}$. Taking into account the $\mathrm{D}$ band temperature shift (Fig. 2, $\partial E_{g} / \partial T=-8.5 \cdot 10^{-5} \mathrm{eV} / \mathrm{K}$ ), it was found that

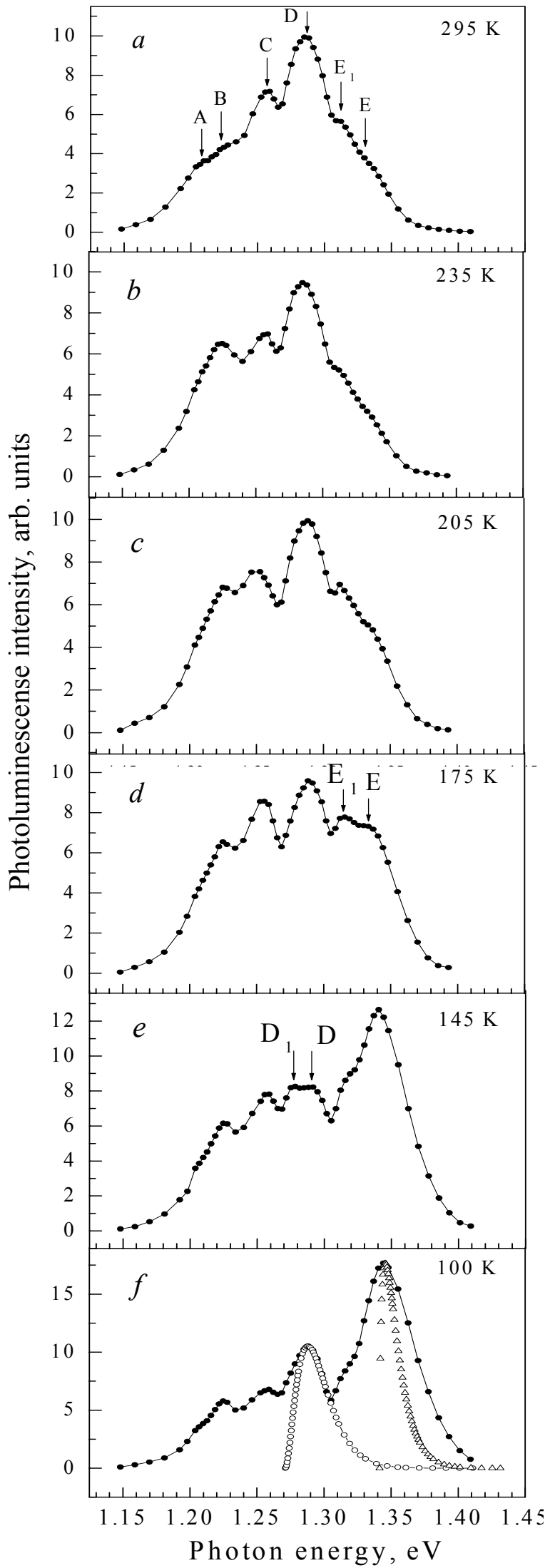

Fig. 1. Photoluminescence spectra of $n$-InSe sample at various temperatures. 
$E_{g}^{i}(295 \mathrm{~K})=1.253 \mathrm{eV}$. The radiative spectra, attributed to the transitions involving the donor $E_{D}$ and acceptor $E_{A}$ levels, are determined by an equation analogous to the equation (1) but with the energy shift by the energy depth of these levels from the corresponding bands. Thus, to determine the $E_{D}$ and $E_{A}$ values it is enough to determine the energy distances between the C-D and BD peaks. The average values of $E_{D}$ and $E_{A}$ measured for several samples are 0.026 and $0.060 \mathrm{eV}$, respectively. According to [13], the photon energy of the radiative donor-acceptor recombination (A band) is described by the expression

$h v=E_{g}^{i}-\left(E_{A}+E_{D}\right)+\frac{e^{2}}{\varepsilon \cdot r}$,

where $e$ is the electron charge, $\varepsilon$ is the dielectric constant of InSe, $r$ is the distance between the donor and acceptor centers. With account of $h v(\mathrm{~A})=1.206 \mathrm{eV}$ and $\varepsilon=7.36$ [14], we have calculated $r=62.3 \mathrm{~nm}$.

The shortwave bands $\mathrm{E}_{1}$ and $\mathrm{E}$ correspond to direct band-to-band transitions. Their intensity sharply increases with lowering temperature whereas that of the $\mathrm{D}$ band one decreases. In the case of direct transitions, the spectrum of intrinsic radiation has a form [12]:

$I(h v) \approx v^{2}\left(h v-E_{g}^{d}\right)^{1 / 2} \exp \left[-\frac{\left(h v-E_{g}^{d}\right)}{k T}\right]$,

where $E_{g}^{d}$ is the direct bandgap. The calculated curve is also shown in Fig. 1f. As one can see, the experimental curve is much broader in comparison to the calculated one. $E_{g}^{d}(100 \mathrm{~K})=1.341 \mathrm{eV}, E_{g}^{d}(295 \mathrm{~K})=1.324 \mathrm{eV}$. Thus, the difference between the $E_{g}^{d}$ and $E_{g}^{i}$ values is $71 \mathrm{meV}$.

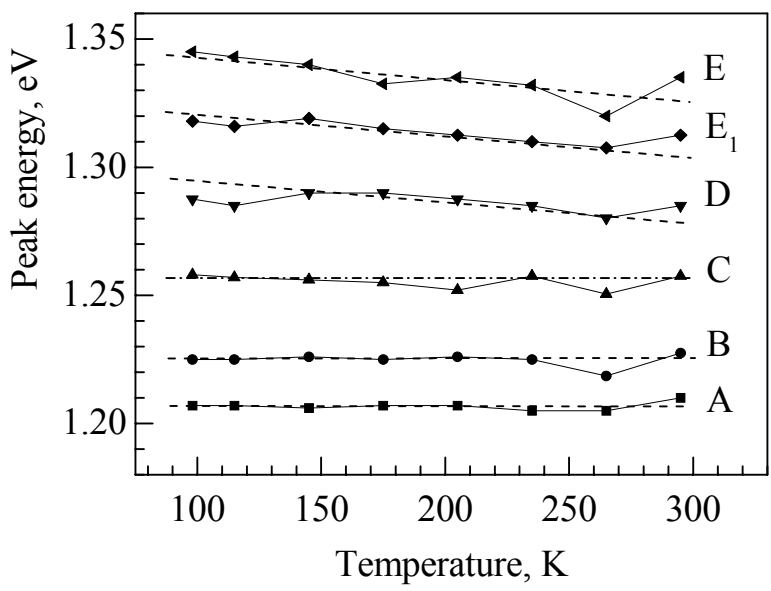

Fig. 2. Temperature shifts of the A, B, C, D, $E_{1}$ and E peaks.

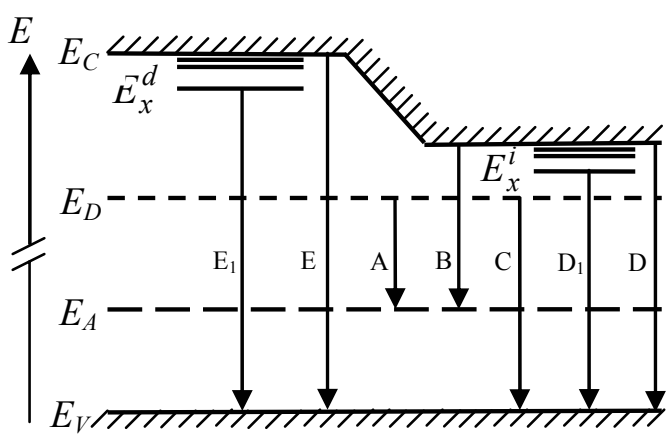

Fig. 3. Energy level diagram of the radiative transitions in InSe.

The temperature dependences of the bands make it possible to establish peculiarities of $\mathrm{D}$ and $\mathrm{E}$ bands. Their doublet character is the best expressed at $145 \mathrm{~K}$ (Fig. 1e) and $175 \mathrm{~K}$ (Fig. 1d). The appearance of $\mathrm{D}_{1}$ and $\mathrm{E}_{1}$ bands is caused by the electron transitions with participation of indirect and direct free excitons from the principal exciton state $n=1$ to the valence band (Fig. 3). Although it is known from the literature about the direct free exciton binding energy $E_{X}^{d}=14.5 \mathrm{meV}$ [15], there are no data for the indirect ones $E_{X}^{i}$. As one can see from the inserts $\mathrm{d}$ and e in Fig. $1, E_{X}^{d}>E_{X}^{i}$. Their values are as follows: $E_{X}^{d}=16.9 \mathrm{meV}$ and $E_{X}^{i}=$ $13.0 \mathrm{meV}$. Note for comparison that the thermal energy $k T$ is equal to 15 and $12.5 \mathrm{meV}$ at 175 and $145 \mathrm{~K}$, respectively. It is less than the binding energy of free excitons at these temperatures and, therefore, allowed to observe the doublet character of D and $\mathrm{E}$ bands. At other temperatures, this peculiarity disappears due to changes in the intensity of the corresponding transitions.

Note that the impurity-bound excitons define the radiative spectra at helium temperatures. Usually, they have weak intensity and, therefore, were not taken into account for interpretation of the obtained spectra.

\section{Conclusions}

PL spectra of undoped InSe were measured within the temperature range of 100 to $295 \mathrm{~K}$. Using comparison of the calculated and experimental spectra, we have carried out an analysis of the radiation bands. It made possible to determine the parameters of energy levels in InSe. We have ascertained that at $T=295 \mathrm{~K}$ the direct and indirect energy gaps are $E_{g}^{d}=1.324 \mathrm{eV}$ and $E_{g}^{i}=1.270 \mathrm{eV}$, respectively. The temperature shift of the bands is $-8.5 \cdot 10^{-5} \mathrm{eV} / \mathrm{K}$. The binding energies of the free direct and indirect excitons are $E_{X}^{d}=16.9 \mathrm{meV}$ and $E_{X}^{i}=$ $13.0 \mathrm{meV}$. The energy depths of the donor level below the conduction band bottom and acceptor level above the valence band top are $E_{D}=26 \mathrm{meV}$ and $E_{A}=60 \mathrm{meV}$, respectively. Distance between the donor and acceptor centers is equal to $62.3 \mathrm{~nm}$. 


\section{References}

1. Abha, A.V.R. Warrier, Photoluminescence studies on the layer semiconductor InSe // J. Appl. Phys. 53(7), p. 5169-5171 (1982).

2. Yu.P. Gnatenko, P.A. Skubenko, Yu.I. Zhirko, O.V. Fialkovskaya, Emission of free and bound excitons in GaSe and InSe crystals in direct and indirect transition region // J. Lumines. 31\&32(1), p. $472-475$ (1984).

3. T. Ikari, S. Shigetomi, Photoluminescence of ntype InSe // Phys. status solidi (b) 124(1), p. K49K51 (1984).

4. J.L. Brebner, T. Steiner, M.L.W. Thewalt, Timeresolved photoluminescence study of InSe // Solid State Communs 56(11), p. 929-931 (1985).

5. J. Riera, A. Segura, A. Chevy, Photoluminescence in silicon-doped n-indium selenide // Phys. status solidi (a) 142(1), p. 265-274 (1994).

6. B. Abay, H. Efeoğlu, Y.K. Yoğurtçu, Lowtemperature photoluminescence of n-InSe layer semiconductor crystals // Mat. Res. Bull. 33(9), p. 1401-1410 (1998).

7. F.J. Manjón, Y. van de Vijver, A. Segura, V. Muñoz, Z.X. Liu, C. Ulrich, Band-to-band and band-to-acceptor photoluminescence studies in InSe under pressure // Phys. status solidi (b) 211, p. $105-110$ (1999).
8. A.A. Homs, B. Mari, Photoluminescence of undoped and neutron-transmutation-doped InSe // J. Appl. Phys. 88(8), p. 4654-4659 (2000).

9. G. Saintogne, J.L. Brebner, Magneto-optical properties of the $\mathrm{Ga}_{x} \mathrm{In}_{1-x} \mathrm{Se}$ system near the fundamental band gap // Phys. Rev.B 30(4), p. 1957-1961 (1984).

10. J. Martınez-Pastor, A. Segura, J.L. Valdes, A. Chevy, Electrical and photovoltaic properties of indium-tin-oxide/p-InSe/Au solar cells // J. Appl. Phys. 62(4), p. 1477-1483 (1987).

11. A. Chevy, A. Kuhn, M.S. Martin, Large InSe monocrystals grown from non-stoichiometric melt // J. Crystal Growth 38(1), p. 118-122 (1977).

12. C.M. Sze, Physics of semiconductor devices. John Wiley and Sons, New York, 1969.

13. A.A. Bergh, P.J. Dean, Light-emitting diodes. Clarendon Press, Oxford, 1976.

14. Landolt-Börnstein, Numerical Data and Functional Relationships in Science and Technology, New Ser. Group III: Crystal and Solid State Physics, 17(f), ed. by O. Madelung. Springer, Berlin e.a. (1983).

15. J. Camassel, P. Merle, H. Mathieu, A. Chevy, Excitonic absorption edge of indium selenide // Phys. Rev. B 17(12), p. 4718-4725 (1978). 